\title{
Real-world costs of autosomal dominant polycystic kidney disease in the Nordics
}

Daniel Eriksson ${ }^{1 *}$, Linda Karlsson ${ }^{1}$, Oskar Eklund ${ }^{1}$, Hans Dieperink², Eero Honkanen ${ }^{3}$, Jan Melin ${ }^{4}$, Kristian Selvig $^{5}$ and Johan Lundberg ${ }^{6}$

\begin{abstract}
Background: There is limited real-world data on the economic burden of patients with autosomal dominant polycystic kidney disease (ADPKD). The objective of this study was to estimate the annual direct and indirect costs of patients with ADPKD by severity of the disease: chronic kidney disease (CKD) stages 1-3; CKD stages 4-5; transplant recipients; and maintenance dialysis patients.

Methods: A retrospective study of ADPKD patients was undertaken April-December 2014 in Denmark, Finland, Norway and Sweden. Data on medical resource utilisation were extracted from medical charts and patients were asked to complete a self-administered questionnaire.

Results: A total of 266 patients were contacted, 243 (91\%) of whom provided consent to participate in the study. Results showed that the economic burden of ADPKD was substantial at all levels of the disease. Lost wages due to reduced productivity were large in absolute terms across all disease strata. Mean total annual costs were highest in dialysis patients, driven by maintenance dialysis care, while the use of immunosuppressants was the main cost component for transplant care. Costs were twice as high in patients with CKD stages 4-5 compared to CKD stages 1-3.

Conclusions: Costs associated with ADPKD are significant and the progression of the disease is associated with an increased frequency and intensity of medical resource utilisation. Interventions that can slow the progression of the disease have the potential to lead to substantial reductions in costs for the treatment of ADPKD.
\end{abstract}

Keywords: Polycystic kidney diseases, ADPKD, Health Care Costs, Health Expenditures, Cost of Illness

\section{Background}

Autosomal dominant polycystic kidney disease (ADPKD) is a dominantly inherited systemic disease characterised by progressive growth of renal cysts. Recent studies in Europe estimate the prevalence at around one in 3000 people [1, 2], equivalent to fewer than 200,000 cases in the European Union. While a rare disease overall, ADPKD is one of the most common hereditary diseases.

Clinical symptoms of renal disease can occur at any age but typically begin in the third or fourth decade of life [3]. Kidney volume growth is due to cyst expansion and precedes functional renal deterioration (as measured by glomerular filtration rate [GFR]) by several decades. Compensatory hyperfiltration in surviving nephrons

\footnotetext{
* Correspondence: d_eriksson@outlook.com

'Quantify Research, Hantverkargatan 8, 11221 Stockholm, Sweden Full list of author information is available at the end of the article
}

initially maintains renal function near normal values. Around $50 \%$ of patients require renal replacement therapy due to kidney failure, which typically develops in the fourth to sixth decade of life [3]. Conventional treatments are tailored to reduce morbidity due to complications of the disease [4]. However, new treatment options slowing down the progression of the disease have now become available [5]. Transplantation is the treatment of choice for end-stage renal disease (ESRD) in ADPKD [4]. Still only a limited number of patients with ESRD undergo transplantation instead of dialysis as initial renal replacement therapy [6].

There is sparse information on the economic burden of ADPKD. One study showed an association between direct medical costs and advanced renal dysfunction in patients with polycystic kidney disease who were free of indications of dialysis or transplantation at baseline [7]. A recent study 
of early-stage ADPKD patients with normal kidney function, found that these patients added a sizable economic burden to the health care system relative to the general population [8]. In a cross-sectional analysis, ADPKD patients, compared to chronic kidney disease (CKD) patients, were found to be younger and generally healthier [9]. However, kidney-related complications and major kidney procedures were more common among ADPKD patients. Further, a retrospective study of medical resource utilisation in ESRD showed that ADPKD patients were younger at dialysis initiation and had lower medical costs compared to control patients with ESRD etiologies other than ADPKD [10].

Cost estimates of ADPKD have been predominantly based on US reimbursement claims data and focused on direct medical resource utilisation for a subset of the population. The objective of this study was to estimate the annual direct and indirect costs of patients with ADPKD, by severity of the disease, in the Nordics.

\section{Methods}

\section{Study design}

This was a cross-sectional study of patients with ADPKD based on data collected from medical charts and patient self-administered questionnaires. Nine nephrology clinics participated; four in Denmark, one in Finland, two in Norway and two in Sweden. Between April and December 2014, we screened and enrolled convenience samples of subjects from each clinic. Patients were recruited by phone or in-person during routine clinical care.

Ethics approvals for the study were granted by the Helsinki University Hospital Ethical Review Board, the Regional Committee of Medical and Health Research Ethics in Oslo (REC South East) and the Regional Ethical Review Board in Stockholm. While the study was reported to the Danish Health and Medicines Authority, formal ethics approval was not required due to the non-interventional design. The study protocol and consent procedures were also reviewed and approved by the participating clinics.

\section{Sample and inclusion criteria}

Subjects were enrolled into four mutually exclusive strata using a hierarchical approach:

1. maintenance dialysis: patients currently on dialysis with or without transplanted kidney

2. transplant recipients: patients with a functioning transplanted kidney, currently not on dialysis

3. CKD stages 4-5: patients not currently on dialysis/ no previous transplant

4. CKD stages 1-3: patients not currently on dialysis/ no previous transplant
Disease severity among ADPKD patients was determined using the estimated GFR (eGFR), as calculated by each respective laboratory; eGFR $<30 \mathrm{ml} / \mathrm{min} / 1.73 \mathrm{~m}^{2}$ for CKD stages $4-5$ and eGFR $\geq 30 \mathrm{ml} / \mathrm{min} / 1.73 \mathrm{~m}^{2}$ for CKD stages $1-3$. The most recent laboratory value was used to establish disease severity at enrolment date. Enrolment of patients was tracked in order to achieve a balanced recruitment across the four groups.

Subjects were eligible for enrolment in the study if they were 18 years of age or older and had been managed for ADPKD at the clinic during the past 12 months. Furthermore, participants were required to have had an eGFR value recorded in the past 12 months (not applicable if on dialysis). Subjects were excluded if they had been involved in a clinical trial in the past 12 months that resulted in a change in the standard of care received. Patients on maintenance dialysis were required to have had initiated dialysis at least six months prior to enrolment. Similarly, patients with a working kidney transplant were required to have had undergone the transplant procedure at least six months prior to enrolment. Finally, informed written consent was required for participation in the study.

\section{Data collection}

Data were extracted from medical charts using a standardised case report form (CRF) and complemented with a self-administered questionnaire [see Additional files 1 and 2]. The CRF and questionnaire were matched for each subject using anonymised subject identifiers.

The standardised CRF allowed for uniform collection of demographic data, disease history and annual ADPKDrelated resource utilisation. The CRF covered the 12month period prior to patient enrolment (enrolment date).

The questionnaire, completed by patients, included complementary questions on ADPKD-related healthcare services received in the past four weeks outside of the primary nephrology clinic, including informal care. Indirect morbidity measures in terms of time missed from work and impairment of work productivity were obtained using the Work Productivity and Activity Impairment (WPAI:GH) questionnaire [11].

\section{Cost estimation}

A societal perspective was used to estimate total costs. We summarised both direct and indirect annual costs related to ADPKD. Resources used in the past 12 months were quantified for each patient and multiplied by unit costs to derive total annual costs. Unit costs for healthcare services (e.g. primary care visit or blood transfusion) were obtained from local and national pricelists as presented in Table 1. Additional costs were derived from public reports and national statistics offices (e.g. daily cost of peritoneal dialysis or gross earnings/employment rates). 
Table 1 Data sources for estimating costs

\begin{tabular}{|c|c|c|}
\hline Type of data & Country & Source \\
\hline \multirow[t]{4}{*}{ Direct costs $^{a}$} & Denmark & $\begin{array}{l}\text { Danish Medicines Agency [13] } \\
\text { Statens Serum Institut [14] } \\
\text { The Capital Region of Denmark [15] } \\
\text { Rigshospitalet [16] }\end{array}$ \\
\hline & Finland & $\begin{array}{l}\text { Pharmaceuticals Pricing Board [17] } \\
\text { The Social Insurance Institution of Finland [18] } \\
\text { The Hospital District of Helsinki and } \\
\text { Uusimaa [19, 20] } \\
\text { National institute for health and welfare [21] } \\
\text { Kuopio University Hospital [22] }\end{array}$ \\
\hline & Norway & $\begin{array}{l}\text { Norwegian Medicines Agency [23] } \\
\text { Norwegian Directorate of Health }[24,25] \\
\text { Ministry of Health and Care Services [26] }\end{array}$ \\
\hline & Sweden & $\begin{array}{l}\text { Dental and Pharmaceutical Benefits } \\
\text { Agency [27] } \\
\text { Swedish Association of the Pharmaceutical } \\
\text { Industry [12] } \\
\text { Region Skåne [28] } \\
\text { Stockholm County Council [29] }\end{array}$ \\
\hline \multirow[t]{4}{*}{ Indirect costs } & Denmark & $\begin{array}{l}\text { Statistics Denmark [30, 31] } \\
\text { Eurostat [32] } \\
\text { KPMG [33] }\end{array}$ \\
\hline & Finland & Statistics Finland $[34,35]$ \\
\hline & Norway & $\begin{array}{l}\text { Statistics Norway [36-38] } \\
\text { KPMG [33] }\end{array}$ \\
\hline & Sweden & $\begin{array}{l}\text { Statistics Sweden }[39,40] \\
\text { Swedish Tax Agency [41] }\end{array}$ \\
\hline
\end{tabular}

${ }^{\mathrm{a}}$ Transportation costs (to and from haemodialysis) were based on answers in the self-administered questionnaire: taxi, $15 \mathrm{~km}$; public transport, $30 \mathrm{~min}$ duration; car, $30 \mathrm{~km}$

Medical resource utilisation was analysed in terms of hospitalisation, outpatient visits, primary care visits, transportation, surgical procedures, diagnostic tests and pharmacotherapy. Pharmacotherapy costs were estimated using conservative dosage estimates as per the drug label [12] for the following classes: antihypertensives, phosphate binders, erythropoiesis-stimulating agents (ESAs), analgesics for kidney pain, vitamin D analogues and immunosuppressive agents.

Indirect costs included informal care and productivity loss. Cost of informal care was based on hours of help from
Table 2 Patient characteristics at enrolment date

\begin{tabular}{|c|c|c|c|c|c|}
\hline $\begin{array}{l}\text { Patient } \\
\text { characteristic }\end{array}$ & $\begin{array}{l}\text { CKD 1-3 } \\
(n=64)\end{array}$ & $\begin{array}{l}\text { CKD 4-5 } \\
(n=55)\end{array}$ & $\begin{array}{l}\text { Dialysis } \\
(\mathrm{n}=61)\end{array}$ & $\begin{array}{l}\text { Transplant } \\
(n=63)\end{array}$ & $P$ value \\
\hline Country, n (\%) & & & & & $<0.0001$ \\
\hline Denmark & $26(41)$ & $32(58)$ & $32(52)$ & $28(44)$ & \\
\hline Sweden & $19(30)$ & $12(22)$ & $14(23)$ & $13(21)$ & \\
\hline Norway & $19(30)$ & $11(20)$ & $4(7)$ & $16(25)$ & \\
\hline Finland & $0(0)$ & $0(0)$ & $11(18)$ & $6(10)$ & \\
\hline Sex (female), n (\%) & $38(59)$ & $29(53)$ & $33(54)$ & $31(49)$ & 0.7144 \\
\hline $\begin{array}{l}\text { Age (years), } \\
\text { mean } \pm S D^{b}\end{array}$ & $52 \pm 13$ & $57 \pm 12$ & $64 \pm 10$ & $59 \pm 10$ & $<0.0001$ \\
\hline $\begin{array}{l}\text { BMI }\left(\geq 30 \mathrm{~kg} / \mathrm{m}^{2}\right) \text {, } \\
\text { n (\%) }\end{array}$ & $10(16)$ & $11(20)$ & $15(25)$ & $14(22)$ & 0.7667 \\
\hline $\begin{array}{l}\text { Currently employed, } \\
\text { n (\%) }\end{array}$ & $40(63)$ & $27(49)$ & $13(21)$ & $28(44)$ & $<0.0001$ \\
\hline $\begin{array}{l}\text { Currently employed } \\
\text { (aged <65 years), } \\
\text { n (\%) }\end{array}$ & $40(78)$ & $27(64)$ & $12(38)$ & $26(59)$ & $<0.0001$ \\
\hline $\begin{array}{l}\text { Comorbidities ( } \geq 1) \text {, } \\
n(\%)\end{array}$ & $43(67)$ & $44(80)$ & $61(100)$ & $45(76)$ & $<0.0001$ \\
\hline $\begin{array}{l}\text { Dialysis in the past } \\
12 \text { months, } \mathrm{n}(\%)^{\mathrm{a}}\end{array}$ & & & $61(100)$ & $5(8)$ & $<0.0001$ \\
\hline Haemodialysis & & . & $51(84)$ & $5(100)$ & 1.0000 \\
\hline Peritoneal dialysis & & & $12(20)$ & $0(0)$ & 0.5754 \\
\hline
\end{tabular}

$P$ values calculated with $X^{2}$ test unless otherwise specified

$S D$ standard deviation, $B M I$ body mass index

${ }^{\text {a } F i s h e r ' s ~ e x a c t ~ t e s t ~}$

${ }^{\mathrm{b}}$ Kruskal-Wallis test

family and friends in the patient's home and calculated using data on average national gross earnings. Productivity loss was estimated using the human capital approach, taking the patient's perspective and counting every lost hour of work as lost production and income [42]. Age- and sexdependent gross earnings and employment rates were obtained from official statistics offices in each country, with employment overheads and benefits added on top. It was assumed that ADPKD patients would have had the same employment rate as the general population had they not been ill.

Table 3 Annual resource utilisation

\begin{tabular}{|c|c|c|c|c|c|}
\hline Mean resource utilisation, past 12 months \pm SD & CKD 1-3 $(n=64)$ & CKD 4-5 $(n=55)$ & Dialysis $(n=61)$ & Transplant $(n=63)$ & $P$ value \\
\hline Number of hospitalisations & $0.2 \pm 0.6$ & $0.5 \pm 1.1$ & $1.8 \pm 2.3$ & $0.6 \pm 1.0$ & $<0.0001$ \\
\hline Number of hospital days & $0.9 \pm 3.1$ & $2.3 \pm 6.9$ & $9.2 \pm 13.6$ & $4.4 \pm 10.2$ & $<0.0001$ \\
\hline Number of hospital days (at least one hospitalisation) & $6.9 \pm 6.5$ & $8.7 \pm 11.6$ & $15.7 \pm 14.7$ & $12.4 \pm 14.1$ & 0.1878 \\
\hline Number of outpatient visits ${ }^{a}$ & $5.2 \pm 10.5$ & $8.2 \pm 17.1$ & $15.2 \pm 24.1$ & $11.6 \pm 13.7$ & $<0.0001$ \\
\hline Number of primary care visits ${ }^{\mathrm{b}}$ & $2.2 \pm 5.5$ & $3.8 \pm 16.8$ & $1.9 \pm 8.0$ & $1.2 \pm 3.8$ & 0.6401 \\
\hline Number of surgical procedures & $0.1 \pm 0.4$ & $0.3 \pm 0.7$ & $1.6 \pm 3.1$ & $0.6 \pm 1.3$ & $<0.0001$ \\
\hline Hours of help: Healthcare professional ${ }^{b}$ & $27.0 \pm 149.8$ & $1.8 \pm 9.6$ & $17.1 \pm 61.2$ & $6.4 \pm 40.4$ & 0.1155 \\
\hline Hours of help: Home care assistant ${ }^{\mathrm{b}}$ & $0.0 \pm 0.0$ & $0.2 \pm 1.8$ & $27.6 \pm 132.6$ & $0.8 \pm 3.8$ & $<0.0001$ \\
\hline Hours of help: Family member or friend ${ }^{b}$ & $3.1 \pm 18.2$ & $27.0 \pm 84.6$ & $104.8 \pm 325.1$ & $11.0 \pm 31.0$ & $<0.0001$ \\
\hline
\end{tabular}

$P$ values calculated with Kruskal-Wallis test

${ }^{a}$ Excluding visits for maintenance dialysis

${ }^{\mathrm{b}}$ Based on the past 4 weeks, self-reported 
Table 4 Annual drug utilisation

\begin{tabular}{|c|c|c|c|c|c|}
\hline $\begin{array}{l}\text { Proportion (\%) of } \\
\text { patients using drug } \\
\text { class, past } 12 \text { months }\end{array}$ & $\begin{array}{l}\text { CKD 1-3 } \\
(n=64)\end{array}$ & $\begin{array}{l}\text { CKD 4-5 } \\
(n=55)\end{array}$ & $\begin{array}{l}\text { Dialysis } \\
(\mathrm{n}=61)\end{array}$ & $\begin{array}{l}\text { Transplant } \\
(\mathrm{n}=63)\end{array}$ & $P$ value \\
\hline Antihypertensives & 84 & 98 & 84 & 87 & 0.0275 \\
\hline Phosphate binders & 0 & 21 & 95 & 14 & $<0.0001$ \\
\hline ESAs & 2 & 13 & 80 & 15 & $<0.0001$ \\
\hline $\begin{array}{l}\text { Analgesics for } \\
\text { kidney pain }\end{array}$ & 17 & 28 & 25 & 17 & 0.4149 \\
\hline Vitamin D analogs ${ }^{\mathrm{a}}$ & 14 & 57 & 97 & 43 & $<0.0001$ \\
\hline Immunosupressants $^{a}$ & 0 & 0 & 7 & 100 & $<0.0001$ \\
\hline Other drugs & 19 & 36 & 90 & 41 & $<0.0001$ \\
\hline
\end{tabular}

$P$ values calculated with Fisher's exact test unless otherwise specified ESA Erythropoiesis-stimulating agent

${ }^{a} X^{2}$ test

Annual cost estimates were derived using national cost data and expressed in the local currency of each respective country (2014 values).

\section{Statistical analyses}

Summary statistics were calculated, including means and standard deviations (SDs) for continuous variables and frequency distributions for categorical variables. We presented costs as means and used non-parametric bootstrapping procedures to derive $95 \%$ confidence intervals.
Table 5 Productivity loss

\begin{tabular}{llllll}
\hline $\begin{array}{l}\text { WPAI-GH } \\
\text { percent } \\
(\%) \pm S D\end{array}$ & $\begin{array}{l}\text { CKD 1-3 } \\
(n=61)\end{array}$ & $\begin{array}{l}\text { CKD 4-5 } \\
(n=53)\end{array}$ & $\begin{array}{l}\text { Dialysis } \\
(n=57)\end{array}$ & $\begin{array}{l}\text { Transplant } \\
(n=63)\end{array}$ & $P$ value \\
\hline $\begin{array}{l}\text { Activity } \\
\text { impairment } \\
\text { due to health }\end{array}$ & $16.7 \pm 24.4$ & $29.4 \pm 28.0$ & $52.6 \pm 27.2$ & $30.4 \pm 27.5$ & $<0.0001$ \\
$\begin{array}{l}\text { Overall work } \\
\text { impairment } \\
\text { due to health }\end{array}$ & $8.7 \pm 14.6$ & $22.8 \pm 28.7$ & $41.8 \pm 33.5$ & $16.4 \pm 23.1$ & 0.0025 \\
$\begin{array}{l}\text { Work time } \\
\text { missed due }\end{array}$ & $4.2 \pm 17.3$ & $8.3 \pm 18.9$ & $25.9 \pm 32.8$ & $4.6 \pm 19.6$ & 0.0014 \\
$\begin{array}{l}\text { to health } \\
\text { (absenteeism) }\end{array}$ & & & & & \\
$\begin{array}{l}\text { Impairment } \\
\text { while working } \\
\text { due to health } \\
\text { (presenteeism) }\end{array}$ & $7.4 \pm 12.2$ & $18.8 \pm 24.1$ & $25.8 \pm 23.9$ & $15.0 \pm 20.8$ & 0.0109 \\
\hline
\end{tabular}

$P$ values calculated with Kruskal-Wallis test

WPAI-GH Work Productivity and Activity Impairment-General Health

a Patients were asked to estimate impairment in the past 7 days (recall period)

Differences across strata were evaluated using the Kruskal-Wallis and $\chi^{2}$ /Fisher's exact tests as appropriate. Resource utilisation in the past four weeks, as captured in the self-administered questionnaire, was extrapolated to one year. Data management and analysis were performed using Stata 12.1 (StataCorp LP, College Station, TX, USA).

Table 6 Annual costs in Danish krone (Denmark)

\begin{tabular}{|c|c|c|c|c|c|}
\hline $\begin{array}{l}\text { Costs in DKK, } \\
\text { mean }(95 \% \mathrm{Cl})\end{array}$ & CKD 1-3 $(n=64)$ & CKD 4-5 $(n=55)$ & Dialysis $(n=61)$ & Transplant $(n=63)$ & $P$ value \\
\hline Direct costs & $28,022(14,728-50,835)$ & $47,203(35,863-63,990)$ & $667,362(623,398-720,640)$ & $196,114(159,055-237,980)$ & $<0.0001$ \\
\hline Hospitalisations & $4736(1611-10,505)$ & $12,224(4810-24,391)$ & $50,954(34,240-71,642)$ & $23,881(12,072-40,151)$ & $<0.0001$ \\
\hline Outpatient care visits & $5596(3810-7867)$ & $9483(6889-14,515)$ & $15,802(11,175-21,341)$ & $14,219(10,992-18,453)$ & $<0.0001$ \\
\hline Primary care visits & $1558(779-2761)$ & $2719(712-7252)$ & $1144(163-2942)$ & $791(317-1741)$ & .5094 \\
\hline Surgical procedures & $2183(125-7519)$ & $6938(2660-13,242)$ & $31,596(18,141-50,394)$ & $4228(1812-7559)$ & $<0.0001$ \\
\hline Diagnostic tests & $1591(1055-2225)$ & $1803(1239-2542)$ & $6081(4851-7544)$ & $3464(2374-5095)$ & $<0.0001$ \\
\hline Home care/medical assistance & $9838(38-28,949)$ & $717(62-1940)$ & $12,399(4417-23,648)$ & $2503(313-7919)$ & .0001 \\
\hline Routine dialysis care & - & - & $441,221(417,652-462,446)$ & $14,377(3783-28,905)$ & $<0.0001$ \\
\hline Haemodialysis transportation & - & - & $41,146(33,117-49,306)$ & $214(0-1068)$ & $<0.0001$ \\
\hline Drug use & $2520(1404-3856)$ & $13,318(9605-17,716)$ & $67,020(57,869-79,028)$ & $132,438(110,082-158,657)$ & $<0.0001$ \\
\hline Antihypertensives & $391(295-507)$ & $476(394-559)$ & $343(272-420)$ & $401(303-534)$ & .0560 \\
\hline Phosphate binders & - & $1351(656-2160)$ & $10,551(8212-13,158)$ & $521(123-1242)$ & $<0.0001$ \\
\hline ESAs & $60(0-245)$ & $3366(1286-5953)$ & $23,281(19,497-26,558)$ & 3449 (1459-6102) & $<0.0001$ \\
\hline Analgesics for kidney pain & $17(5-39)$ & $90(21-253)$ & $182(26-534)$ & $13(2-37)$ & .1069 \\
\hline Vitamin D analogues & 2004 (937-3379) & $6955(5201-8861)$ & $13,309(12,162-14,207)$ & $4593(3129-6257)$ & $<0.0001$ \\
\hline Immunosupressants & - & - & $5699(312-14,111)$ & $122,984(100,943-149,412)$ & $<0.0001$ \\
\hline Other drugs & $48(11-110)$ & $1081(157-2500)$ & $13,655(10,825-16,513)$ & 477 (194-932) & $<0.0001$ \\
\hline Indirect costs & $51,523(32,278-75,631)$ & $94,631(65,117-126,721)$ & $100,970(67,789-132,323)$ & $81,688(55,334-110,676)$ & .0726 \\
\hline Productivity loss & $51,224(31,835-75,332)$ & $92,083(63,079-123,547)$ & $91,373(59,420-122,164)$ & $80,647(54,460-109,503)$ & .3032 \\
\hline Informal care & $299(0-896)$ & $2548(873-5141)$ & $9597(3801-19,465)$ & $1041(415-1891)$ & $<0.0001$ \\
\hline Total costs & $79,544(54,826-109,204)$ & $141,834(105,601-181,449)$ & $768,332(707,301-830,831)$ & $277,802(227,251-333,023)$ & $<0.0001$ \\
\hline
\end{tabular}

$P$ values calculated with Kruskal-Wallis test

$D K K$ Danish krone, ESA erythropoiesis-stimulating agent, $C l$ confidence interval (bias corrected) 


\section{Results}

\section{Demographic and clinical characteristics}

A total of 266 patients were contacted. Of these 243 (91\%) provided consent to participate and were enrolled into the four disease strata: CKD stages $1-3(n=64)$, CKD stages $4-5(n=55)$, transplant $(n=61)$, and dialysis $(n=63)$. Overall, 241 (99\%) of participants completed the questionnaire.

Dialysis and transplant patients tended to be older than patients in earlier stages of the disease; those younger than 65 years were $80 \%$ in patients with CKD stages $1-3,76 \%$ in CKD stages $4-5,54 \%$ in dialysis patients and $70 \%$ in transplant recipients. Mean age for initiation of dialysis was 59 years in the dialysis stratum and the average age at the time of kidney transplantation was 52 years. Among those on dialysis, only two patients (3\%) had received both haemodialysis and peritoneal dialysis in the past 12 months. No differences between disease strata were seen in sex and BMI (Table 2). Employment rates were lowest in the dialysis stratum (21\%), with corresponding rates of $44 \%$ in transplant recipients, $49 \%$ in CKD stages $4-5$ and $63 \%$ in CKD stages 1-3.

\section{Medical resource utilisation}

Medical resource utilisation differed substantially between disease strata (Table 3). In general, dialysis patients had the highest number of hospitalisations and outpatient visits, followed by transplant recipients and other dialysisindependent patients. This difference, however, was not observed for primary care visits, as reported in the selfadministered questionnaire.

Only $8 \%$ of CKD stages $1-3$ patients had a surgery related to ADPKD in the past year, compared to $18 \%$ of CKD stages $4-5$ patients, $29 \%$ of transplant recipients and $49 \%$ of dialysis patients. Consequently, there was a significant difference in the mean number of surgical procedures in the past year between the disease strata, ranging from 0.1 in patients with CKD stages $1-3$ to 1.6 in dialysis patients. Among transplant recipients, $10 \%$ had received the transplant in the past year. Similarly, 25\% of dialysis patients had initiated treatment in the past year.

Dialysis patients were generally prescribed more drugs compared to the other disease states; $95 \%$ of dialysis patients used phosphate binders, $80 \%$ used erythropoiesisstimulating agents (ESAs) and 97\% were prescribed vitamin D analogues (Table 4). Analgesics for kidney pain were, however, most common in CKD stages 4-5, used by $27 \%$ compared to $16-23 \%$ in the other disease strata. Almost all patients with CKD stages 4-5 (98\%) were prescribed antihypertensives.

Among dialysis patients $59 \%$ travelled by taxi to receive their treatment, while $35 \%$ drove and $6 \%$ used

Table 7 Annual costs in euro (Finland)

\begin{tabular}{|c|c|c|c|c|c|}
\hline Costs in EUR, mean $(95 \% \mathrm{Cl})$ & CKD 1-3 $(n=64)$ & CKD 4-5 ( $n=55)$ & Dialysis $(n=61)$ & Transplant $(n=63)$ & $P$ value \\
\hline Direct costs & 3676 (2223-6190) & $5883(4588-7701)$ & $64,811(60,460-70,417)$ & $20,305(16,228-25,166)$ & $<0.0001$ \\
\hline Hospitalisations & $507(172-1125)$ & $1309(515-2611)$ & $5455(3666-7670)$ & 2557 (1283-4299) & $<0.0001$ \\
\hline Outpatient care visits & $1159(843-1569)$ & $2057(1606-2871)$ & $3203(2329-4230)$ & $3197(2502-4155)$ & $<0.0001$ \\
\hline Primary care visits & $237(117-403)$ & $414(84-1097)$ & $174(50-548)$ & $121(48-265)$ & .5094 \\
\hline Surgical procedures & $249(15-794)$ & $511(154-1098)$ & $2562(1211-5119)$ & $1009(435-1956)$ & $<0.0001$ \\
\hline Diagnostic tests & $191(125-263)$ & $237(166-321)$ & $659(511-843)$ & $354(251-496)$ & $<0.0001$ \\
\hline Home care/medical assistance & $1048(4-3049)$ & $74(5-204)$ & 1093 (384-2044) & $260(28-837)$ & .0002 \\
\hline Routine dialysis care & - & - & $42,900(40,609-44,964)$ & $1398(368-2810)$ & $<0.0001$ \\
\hline Haemodialysis transportation & - & - & 4090 (3184-5080) & $13(0-25)$ & $<0.0001$ \\
\hline Drug use & $284(175-419)$ & $1281(1008-1600)$ & $4675(4040-5512)$ & $11,396(9404-13,944)$ & $<0.0001$ \\
\hline Antihypertensives & $68(54-86)$ & $113(95-132)$ & $73(60-86)$ & $96(78-117)$ & .0020 \\
\hline Phosphate binders & - & $225(103-362)$ & $1266(1006-1554)$ & $65(15-148)$ & $<0.0001$ \\
\hline ESAs & $4(0-15)$ & $204(78-361)$ & $1413(1181-1611)$ & $209(89-370)$ & $<0.0001$ \\
\hline Analgesics for kidney pain & $3(1-7)$ & $14(5-30)$ & $12(4-25)$ & $4(0-12)$ & .1329 \\
\hline Vitamin D analogues & $198(93-335)$ & $689(516-878)$ & $1318(1207-1407)$ & $455(310-620)$ & $<0.0001$ \\
\hline Immunosupressants & - & - & $440(26-1076)$ & $10,469(8516-12,955)$ & $<0.0001$ \\
\hline Other drugs & $10(3-27)$ & $36(17-61)$ & $154(126-183)$ & $99(48-171)$ & $<0.0001$ \\
\hline Indirect costs & 4863 (2986-7132) & $9904(6738-13,319)$ & $7674(5195-10,042)$ & $7585(5125-10,494)$ & .0925 \\
\hline Productivity loss & 4835 (2959-7104) & $9667(6586-13,018)$ & 6783 (4586-8815) & $7488(5058-10,382)$ & .2742 \\
\hline Informal care & $28(0-83)$ & $237(79-475)$ & $891(353-1807)$ & $97(38-176)$ & $<0.0001$ \\
\hline Total costs & $8539(6042-11,631)$ & $15,787(12,006-20,008)$ & $72,486(67,053-79,025)$ & $27,890(22,669-33,722)$ & $<0.0001$ \\
\hline
\end{tabular}

$P$ values calculated with Kruskal-Wallis test

EUR euro, ESA erythropoiesis-stimulating agent, $\mathrm{Cl}$ confidence interval (bias corrected) 
public transport. Forty-three percent travelled for at least $30 \mathrm{~min}$ one-way to receive treatment.

\section{Activity and work impairment}

The levels of general daily activity impairment and productivity impairment due to health problems differed with disease severity. Activity impairment was highest among dialysis patients with $53 \%$ but also substantial at $30 \%$ in both patients with CKD stages 4-5 and among transplant recipients (Table 5). Among those employed, an average of $4-26 \%$ of work time was missed due to health problems, while patients estimated 7-26\% of time lost while at work, depending on disease severity. Taken together, overall work impairment due to health was significantly different between disease strata. Work impairment was highest among dialysis patients (42\%), followed by CKD stages 4-5 (23\%), transplant recipients (16\%) and CKD stages 1-3 (9\%).

\section{Annual costs associated with ADPKD}

Costs are presented by disease severity and expressed in each respective local currency (Tables 6, 7, 8 and 9). Average total annual costs were highest for dialysis patients, followed by transplant recipients, patients in CKD stages $4-5$ and CKD stages $1-3(\mathrm{P}<0.0001$, for all countries). Compared to CKD stages $1-3$, annual costs were almost twice as high in CKD stages 4-5, two to three times higher in transplant recipients, and seven to nine times higher in dialysis patients. Differences between disease strata were even more pronounced when looking at direct costs alone ( $\mathrm{P}<0.0001$, for all countries). Direct costs were almost twice as high in patients with CKD stages 4-5 compared to stages 1-3, but around six times higher among transplant recipients and 21 times higher among dialysis patients. Direct medical costs were substantial among dialysis patients, with routine dialysis care alone accounting over half of total costs. Productivity loss was a driver of costs across all stages of ADPKD, and especially substantial at around two-thirds of total costs in patients with CKD stages $1-3$ and $4-5$.

\section{Discussion}

In this study we enrolled 243 ADPKD patients from nine nephrology clinics in Denmark, Finland, Norway and Sweden. For these patients we collected and analysed data from medical charts and self-administered questionnaires. Our findings showed that the economic burden of ADPKD was substantial at all levels of disease and that progression of ADPKD was associated with an increased frequency and intensity of medical resource utilisation.

Table 8 Annual costs in Norwegian krone (Norway)

\begin{tabular}{|c|c|c|c|c|c|}
\hline Costs in NOK, mean $(95 \% \mathrm{Cl})$ & CKD 1-3 $(n=64)$ & CKD 4-5 (n = 55) & Dialysis $(n=61)$ & Transplant $(n=63)$ & $P$ value \\
\hline Direct costs & $38,676(18,712-69,343)$ & $80,145(51,159-118,538)$ & $851,277(765,334-959,286)$ & $185,108(131,915-251,557)$ & $<0.0001$ \\
\hline Hospitalisations & $12,898(4387-28,610)$ & $33,291(13,098-66,704)$ & $138,766(93,249-195,108)$ & $65,036(32,637-109,347)$ & $<0.0001$ \\
\hline Outpatient care visits & 4425 (3355-5859) & $8085(6588-10,612)$ & $12,050(8956-15,673)$ & $12,840(10,094-16,706)$ & $<0.0001$ \\
\hline Primary care visits & $691(345-1224)$ & $1205(246-3192)$ & $507(145-1594)$ & $351(140-772)$ & .5094 \\
\hline Surgical procedures & $4209(89-15,018)$ & $23,660(10,359-40,439)$ & $106,888(67,415-153,360)$ & $6798(1790-14,265)$ & $<0.0001$ \\
\hline Diagnostic tests & $1652(1100-2273)$ & $1959(1417-2622)$ & $6423(5132-8002)$ & $3359(2450-4587)$ & $<0.0001$ \\
\hline Home care/medical assistance & $12,317(48-36,245)$ & $885(66-2396)$ & $14,151(5074-27,170)$ & 3090 (359-9872) & .0002 \\
\hline Routine dialysis care & - & - & $495,052(468,607-518,867)$ & $16,131(4245-32,431)$ & $<0.0001$ \\
\hline Haemodialysis transportation & - & - & $32,460(25,631-39,573)$ & $128(0-256)$ & $<0.0001$ \\
\hline Drug use & $2483(1472-3707)$ & $11,060(8445-14,068)$ & $44,980(39,925-51,304)$ & $77,375(64,093-95,936)$ & $<0.0001$ \\
\hline Antihypertensives & $530(435-640)$ & $715(621-808)$ & $522(433-617)$ & $567(461-687)$ & .02459 \\
\hline Phosphate binders & - & $1158(565-1850)$ & 8072 (6345-9932) & $418(102-944)$ & $<0.0001$ \\
\hline ESAs & $36(0-148)$ & $2036(778-3600)$ & $14,079(11,772-16,055)$ & $2086(827-3610)$ & $<0.0001$ \\
\hline Analgesics for kidney pain & $16(6-31)$ & $103(33-251)$ & $104(29-256)$ & $33(3-98)$ & .1053 \\
\hline Vitamin D analogues & $1833(857-3090)$ & $6360(4761-8104)$ & $12,170(11,121-12,991)$ & $4200(2861-5722)$ & $<0.0001$ \\
\hline Immunosupressants & - & - & $2741(290-6569)$ & $69,324(56,582-87,711)$ & $<0.0001$ \\
\hline Other drugs & $68(19-164)$ & $688(181-1422)$ & $7292(5804-8788)$ & $746(353-1326)$ & $<0.0001$ \\
\hline Indirect costs & $111,441(70,268-157,539)$ & $204,324(143,043-268,451)$ & $215,588(144,283-280,047)$ & $182,164(125,099-242,812)$ & .0604 \\
\hline Productivity loss & $110,892(70,085-157,480)$ & $199,644(139,793-263,028)$ & $197,961(129,783-259,106)$ & $180,251(123,425-241,038)$ & .2452 \\
\hline Informal care & $548(0-1645)$ & $4680(1603-9442)$ & $17,627(7001-35,752)$ & $1913(761-3473)$ & $<0.0001$ \\
\hline Total costs & $150,117(104,759-202,958)$ & $284,469(206,680-373,107)$ & $1,066,865(950,458-1,204,094)$ & $367,272(278,949-466,269)$ & $<0.0001$ \\
\hline
\end{tabular}

$P$ values calculated with Kruskal-Wallis test

NOK Norwegian krone, ESA erythropoiesis-stimulating agent, $\mathrm{Cl}$ confidence interval (bias corrected) 
Table 9 Annual costs in Swedish krona (Sweden)

\begin{tabular}{|c|c|c|c|c|c|}
\hline Costs in SEK, mean (95\% Cl) & CKD 1-3 $(n=64)$ & CKD 4-5 $(n=55)$ & Dialysis $(n=61)$ & Transplant $(n=63)$ & $P$ value \\
\hline Direct costs & $28,820(16,123-50,689)$ & $48,624(36,718-65,151)$ & $712,482(668,060-766,530)$ & $173,199(135,833-218,165)$ & $<0.0001$ \\
\hline Hospitalisations & $3812(1297-8456)$ & $9840(3871-19,716)$ & $41,015(27,561-57,668)$ & $19,223(9717-32,319)$ & $<0.0001$ \\
\hline Outpatient care visits & $5878(4291-7943)$ & $10,457(8193-14,512)$ & $16,221(11871-21,402)$ & $16,288(12,728-21,120)$ & $<0.0001$ \\
\hline Primary care visits & $1178(589-2088)$ & $2056(539-5484)$ & $865(124-2225)$ & $598(120-1077)$ & .5094 \\
\hline Surgical procedures & $3349(187-10,713)$ & $7135(1620-15,769)$ & $31,431(16,065-51,975)$ & $8131(3603-14,557)$ & $<0.0001$ \\
\hline Diagnostic tests & $2847(2244-3509)$ & $5810(4535-7332)$ & $25,140(21,308-29,294)$ & 10,987 (8119-14,700) & $<0.0001$ \\
\hline Home care/medical assistance & $9442(37-27,461)$ & $675(47-1850)$ & $10,525(3734-20,134)$ & $2359(263-7537)$ & .0002 \\
\hline Routine dialysis care & - & - & $488,009(461940-511,484)$ & $15,901(4185-32,472)$ & $<0.0001$ \\
\hline Haemodialysis transportation & - & - & $37,145(30,444-43,657)$ & $269(0-1344)$ & $<0.0001$ \\
\hline Drug use & $2313(1333-3490)$ & $12,651(9110-16,825)$ & $62,131(54,647-71,169)$ & $99,443(80,719-125,735)$ & $<0.0001$ \\
\hline Antihypertensives & $419(341-513)$ & $666(556-784)$ & $503(415-603)$ & $583(451-756)$ & .0282 \\
\hline Phosphate binders & - & $1244(598-1996)$ & $9788(7577-12,262)$ & $472(108-1151)$ & $<0.0001$ \\
\hline ESAs & $63(0-257)$ & $3535(1350-6252)$ & $24,450(20,476-27,892)$ & $3622(1533-6409)$ & $<0.0001$ \\
\hline Analgesics for kidney pain & $14(5-26)$ & $102(28-255)$ & $78(21-193)$ & $15(2-37)$ & .1032 \\
\hline Vitamin D analogues & 1776 (824-2952) & $6162(4613-7852)$ & $11,792(10,796-12,592)$ & $4069(2756-5544)$ & $<0.0001$ \\
\hline Immunosupressants & - & - & 3579 (291-8847) & $90,205(72,049-117,127)$ & $<0.0001$ \\
\hline Other drugs & $41(10-95)$ & $942(135-2184)$ & $11,941(9447-14,436)$ & $477(202-909)$ & $<0.0001$ \\
\hline Indirect costs & $64,259(39,484-92,072)$ & $128,541(90,007-169,626)$ & $124,957(85,289-162,184)$ & $112,688(77,160-150,663)$ & .0438 \\
\hline Productivity loss & $63,963(39,446-91,997)$ & $126,019(88,140-165,959)$ & $115,458(77,012-150,462)$ & $111,658(75,795-149,420)$ & .1842 \\
\hline Informal care & $296(0-887)$ & $2522(864-5088)$ & $9499(3762-19,266)$ & $1031(410-1871)$ & $<0.0001$ \\
\hline Total costs & $93,079(64,756-125,857)$ & $177,165(131,147-227,131)$ & $837,438(771,457-903,231)$ & $285,887(228,017-352,229)$ & $<0.000$ \\
\hline
\end{tabular}

$P$ values calculated with Kruskal-Wallis test

SEK Swedish krona, ESA erythropoiesis-stimulating agent, $C l$ confidence interval (bias corrected)

Mean total direct and indirect costs were approximately twice as high in patients with CKD stages 4-5 compared to CKD stages 1-3. Resource utilisation increased substantially as patients progressed to ESRD, with costs among dialysis patients greatly exceeding that of kidney transplant recipients. The use of immunosuppressants accounted for around half of costs in transplant recipients. Similarly, maintenance dialysis care alone accounted for over half of total costs in dialysis patients, who had the highest number of hospitalisations and outpatient visits. Primary care visits were more frequent in earlier stages of the disease. Lost wages due to reduced productivity were large in absolute terms across all disease strata. General daily activity impairment due to health was highest among dialysis patients who reported an average reduction in activity of over $50 \%$. Activity impairment was also substantial in transplant recipients and in patients with CKD stages $4-5$, both at around $30 \%$.

Some limitations of our study should be noted. Selection bias may be an issue as with any observational study. No randomisation was performed and primarily patients who actively sought health care were included. Not all patients in earlier stages of the disease are followed by nephrology clinics and the study design limited the inclusion of transplant recipients to those with a functioning transplant, thus potentially underestimating costs in patients with advanced disease. A proportion of patients with ESRD initiated treatment within 12 months of the enrolment date; however, sensitivity analyses revealed an insignificant impact on mean total costs.

Our study adds to the limited and fragmented literature on cost estimates of ADPKD. To our knowledge this is the first study to provide cost data on an ADPKD population that includes both early stages of the disease, stratified by renal function, and patients with ESRD. A further strength of this study is the enrolment of patients with physician-confirmed diagnosis of ADPKD. Furthermore, in addition to data extraction from medical charts, a self-administered questionnaire, including the WPAI:GH, was used to capture resource utilisation outside of the nephrology clinic and to estimate indirect costs in terms of productivity loss and caregiver support. Finally, we achieved a high response rate with $91 \%$ of invited patients agreeing to participate in the study.

\section{Conclusions}

We provide a thorough description of the medical resource utilisation and costs associated with ADPKD across all stages of the disease. Our findings confirm the association between economic burden and progression of ADPKD [7]. Costs were highest in dialysis patients, driven by maintenance dialysis care, while the use of immunosuppressants 
was the main cost component for transplant care. Costs were twice as high in patients with CKD stages 4-5 compared to CKD stages $1-3$. Consequently, interventions that can slow the progression of the disease have the potential to lead to substantial reductions in costs for the treatment of ADPKD.

\section{Additional files}

Additional file 1: QR ADPKD CRF 20140331 Final v1.1.pdf - Case report form (CRF) - Questionnaire used to collect data from patients' medical charts. (PDF $624 \mathrm{~kb}$ )

Additional file 2: QR ADPKD Additional Questions 20140205 Eng.pdf Self-administered questionnaire - Questionnaire administered to patients. (PDF $16 \mathrm{~kb})$

\section{Abbreviations}

ADPKD: Autosomal dominant polycystic kidney disease; BMl: Body mass index; CKD: Chronic kidney disease; CRF: Case report form; eGFR: Estimated glomerular filtration rate; ESA: Erythropoiesis-stimulating agents; ESRD: Endstage renal disease; SD: Standard deviation; WPAI: Work productivity and activity impairment

\section{Acknowledgements}

The authors would like to thank investigators and research staff for their contribution in patient recruitment and data collection. Specifically we would like to acknowledge Henrik Birn, Aarhus University Hospital; Astrid Dale, Førde Central Hospital; Martin Egfjord and Anne-Lise Kamper, Rigshospitalet; Jeppe HagstrupChristensen, Aalborg University Hospital; and Hans Herlitz, Sahlgrenska University Hospital. We would also like to thank Martin Gisby and Paul Robinson at Otsuka Pharmaceuticals Europe Limited and Anders Gustavsson, Quantify Research, for their valuable input. Finally we thank all patients for their participation in the study.

\section{Funding}

The authors declare that the study was sponsored by Otsuka Pharma Scandinavia.

\section{Availability of data and materials}

The authors declare that the datasets generated and/or analysed during the current study are not publicly available due personal data directives governing handling of sensitive personal data in the European Union. Medical chart data are owned by the respective study site institutions. Release of study data is therefore not possible.

\section{Authors' contributions}

DE designed the study. DE, LK and OE planned and executed the study and prepared the draft manuscript. $\mathrm{HD}, \mathrm{EH}, \mathrm{JM}$ and $\mathrm{KS}$ provided clinical expertise and critically reviewed study documents as well as assisted in data collection at the respective study sites. HD, EH, JM, KS and JL made substantial contributions to the analysis and interpretation of data. All authors critically revised the draft manuscript and approved the final version.

\section{Ethics approval and consent to participate}

Ethics approvals for the study were granted by the Helsinki University Hospital Ethical Review Board, the Regional Committee of Medical and Health Research Ethics in Oslo (REC South East) and the Regional Ethical Review Board in Stockholm. While the study was reported to the Danish Health and Medicines Authority, formal ethics approval was not required due to the non-interventional design. The study protocol and consent procedures were also reviewed and approved by the clinics. All patients gave their informed consent to participate in the study.

\section{Consent for publication}

Not applicable.

\section{Competing interests}

The study was sponsored by Otsuka Pharma Scandinavia. DE, LK and OE are employees of Quantify Research, which has received funds from Otsuka Pharma Scandinavia in connection with this study. HD, EH, JM and KS are members of an advisory board on ADPKD sponsored by Otsuka Pharma Scandinavia. JM has received lecturing fees from Otsuka Pharma Scandinavia. $J \mathrm{~L}$ is an employee of Otsuka Pharma Scandinavia. The manuscript is not under consideration for publication elsewhere in a similar form, in any language, except in abstract form.

\section{Publisher's Note}

Springer Nature remains neutral with regard to jurisdictional claims in published maps and institutional affiliations.

\section{Author details}

${ }^{1}$ Quantify Research, Hantverkargatan 8, 11221 Stockholm, Sweden. ${ }^{2}$ Odense University Hospital, Department of Nephrology, Sdr. Boulevard 29, DK-5000 Odense C, Denmark. ${ }^{3}$ Helsinki University Central Hospital, Department of Medicine, Division of Nephrology, Haartmaninkatu 4, P.O Box 372, FIN-00029 HUS Helsinki, Finland. ${ }^{4}$ Uppsala University Hospital, Department of Nephrology, 75185 Uppsala, Sweden. ${ }^{5}$ Vestre Viken Hospital Trust, Department of Nephrology, Postboks 800 3004, Drammen, Norway. ${ }^{6}$ Otsuka Pharma Scandinavia, Birger Jarlsgatan 27, 11145 Stockholm, Sweden.

Received: 19 October 2016 Accepted: 7 August 2017 Published online: 15 August 2017

\section{References}

1. McGovern AP, Jones S, van Vlymen J, Saggar AK, Sandford R, de Lusignan S Identification of people with autosomal dominant polycystic kidney disease using routine data: a cross sectional study. BMC nephrology. 2014;15(1):182

2. Neumann HP, Jilg C, Bacher J, Nabulsi Z, Malinoc A, Hummel B, Hoffmann MM, Ortiz-Bruechle N, Glasker S, Pisarski P. Epidemiology of autosomaldominant polycystic kidney disease: an in-depth clinical study for southwestern Germany. Nephrology Dialysis Transplantation. 2013;28(6):1472-87.

3. Grantham JJ. Autosomal dominant polycystic kidney disease. New England Journal of Medicine. 2008:359(14):1477-85.

4. Torres VE, Harris PC, Pirson Y. Autosomal dominant polycystic kidney disease. Lancet. 2007;369(9569):1287-301.

5. Torres VE, Chapman AB, Devuyst O, Gansevoort RT, Grantham JJ, Higashihara E, Perrone RD, Krasa HB, Ouyang J, Czerwiec FS. Tolvaptan in patients with autosomal dominant polycystic kidney disease. New England Journal of Medicine. 2012;367(25):2407-18

6. Abecassis M, Bartlett ST, Collins AJ, Davis CL, Delmonico FL, Friedewald JJ, Hays R, Howard A, Jones E, Leichtman AB, et al. Kidney transplantation as primary therapy for end-stage renal disease: a National Kidney Foundation/Kidney Disease Outcomes Quality Initiative (NKF/KDOQITM) conference. Clinical journal of the American Society of Nephrology : CJASN. 2008:3(2):471-80.

7. Lentine KL, Xiao H, Machnicki G, Gheorghian A, Schnitzler MA: Renal function and healthcare costs in patients with polycystic kidney disease. Clin J Am Soc Nephrol. 2010:CJN. 00780110.

8. Knight T, Schaefer C, Krasa H, Oberdhan D, Chapman A, Perrone RD. Medical resource utilization and costs associated with autosomal dominant polycystic kidney disease in the Usa: a retrospective matched cohort analysis of private insurer data. ClinicoEconomics and outcomes research: CEOR. 2015;7:123.

9. Blanchette CM, Craver C, Belk KW, Lubeck DP, Rossetti S, Gutierrez B. Hospital-based inpatient resource utilization associated with autosomal dominant polycystic kidney disease in the US. Journal of medical economics. 2015;18(4):303-11.

10. Brunelli SM, Blanchette CM, Claxton AJ, Roy D, Rossetti S, Gutierrez B. Endstage renal disease in autosomal dominant polycystic kidney disease: a comparison of dialysis-related utilization and costs with other chronic kidney diseases. ClinicoEconomics and outcomes research: CEOR. 2015;7:65.

11. Reilly MC, Zbrozek AS, Dukes EM. The validity and reproducibility of a work productivity and activity impairment instrument. Pharmacoeconomics. 1993;4(5):353-65.

12. FASS Vårdpersonal. www.fass.se. Accessed 15 Dec 2014

13. Medicinpriser.dk. www.medicinpriser.dk. Accessed 15 Jan 2015. 
14. Takstsystem, Vejledning. https:/ny.sundhedsdatastyrelsen.dk/-/media/sds/ filer/finansiering-og-afregning/takster/2015/takstvejledning2015.pdf. Accessed 15 Mar 2015.

15. Takstkatalog for højt specialiseret behandling i Region Hovedstaden. [https://www.regionh.dk/om-region-hovedstaden/oekonomi/Regionensoekonomi-og-finansiering/Publishinglmages/Sider/Regionens-budget-ogoekonomi/Takstkatalog2012.pdf. Accessed 15 March, 2015

16. Klinisk biokemisk afdeling: metodeliste. labportal.rh.dk. Accessed 15 Mar 2015

17. Reimbursable authorized medicinal products and their prices. http://www. hila.fi/fi/tiedotteet-ja-luettelot/luettelot/korvattavat-myyntiluvallisetlaakevalmisteet. Accessed 15 Jan 2016.

18. Kela Medicinal Products Database. https://easiointi.kela.fi/laakekys_app/ LaakekysApplication?kieli=fi. Accessed 15 Jan 2016.

19. Palveluhinnasto 2015 - Osa 1 Tuotteistetut sairaanhoidolliset palvelut. http://www.hus.fi/hus-tietoa/talous/Hinnoittelu/Documents/ HUS\%20Palveluhinnasto\%202015\%200SA\%201.pdf. Accessed 15 Jan 2016.

20. Palveluhinnasto 2015 - Osa 2 suoriteperusteiset sairaanhoidolliset palvelut http://www.hus.fi/hus-tietoa/talous/Hinnoittelu/Documents/ HUS\%20Palveluhinnasto\%202015\%200SA\%202.pdf. Accessed 15 Jan 2016.

21. Terveyden- ja sosiaalihuollon yksikkökustannukset Suomessa vuonna. 2011. https://www.julkari.fi/handle/10024/114683. Accessed 15 Jan 2016.

22. Kliinisten erikoisalojen palvelutuotteet, suoritteeet ja hinnat. 2016 https://www.psshp.fi/documents/11427/38559/Klinikkahinnasto+2016.pdf. Accessed 15 Jan 2016.

23. NoMA medicine database. www.legemiddelsok.no. Accessed 15 Aug 2015.

24. SAMDATA spesialisthelsetienesten. 2013. mww.helsedir.no. Accessed 15 Aug 2015.

25. Kostnadsvekter og kostnad per pasient (KPP). www.helsedir.no. Accessed 15 Aug 2015

26. Forskrift om godtgjørelse av utgifter til helsehjelp som utføres poliklinisk ved statlige helseinstitusjoner og ved helseinstitusjoner som mottar driftstilskudd fra regionale helseforetak. https://lovdata.no/dokument/SF/ forskrift/2007-12-19-1761. Accessed 15 Aug 2015.

27. TLV Database. www.tlv.se/beslut/. Accessed 15 Dec 2014.

28. Regionala priser och ersättningar för Södra sjukvårdsregionen (2014). Södra Regionvårdsnämnden [www.skane.se/sv/Webbplatser/Sodraregionvardsnamnden/]. Accessed 15 August, 2015

29. Utvecklingsplan för njurdialysvården i stockholms län år 2009-2012. Hälso och sjukvårdsnämnden [sammantradeshandlingar.sll.se]. Accessed 15 Dec, 2014

30. StatBank Denmark. 2013. www.statistikbanken.dk. Accessed 15 Jan 2015.

31. Consumer price index. http://www.dst.dk/da/Statistik/emner/priser-ogforbrug/forbrugerpriser. Accessed 15 Aug 2015.

32. Taxation trends in the European Union: Data for the EU Member States, Iceland and Norway. http://ec.europa.eu/eurostat. Accessed 15 Aug 2015.

33. Tax Rates Online: Indirect tax rates for 2009-2015. https://home.kpmg.com/ xx/en/home/services/tax/tax-tools-and-resources/tax-rates-online/corporatetax-rates-table.html. Accessed 15 Aug 2015.

34. Labour Force Survey. 2013. [http://www.stat.fi/til/tyti/index_en.html]. Accessed 15 Jan 2016

35. PX-Web databases. [http://stat.fi/tup/tilastotietokannat/index_en.html]. Accessed 15 Janu 2016.

36. Consumer price index. www.ssb.no/priser-og-prisindekser/statistikker/kpi. Accessed 14 Aug 2015.

37. Labour Force Survey. 2014. https://www.ssb.no/en/arbeid-og-lonn. Accessed 14 Aug 2015

38. StatBank Norway. 2014. www.ssb.no/statistikkbanken. Accessed 14 Aug 2015.

39. Statistical Database. 2013. www.statistikdatabasen.scb.se. Accessed 15 Dec 2014

40. Labour Force Surveys. 2014. http.//www.scb.se/AM0401-en. Accessed 15 Dec 2014.

41. Arbetsgivaravgifter. 2014. www.skatteverket.se/foretagorganisationer/ arbetsgivare/socialavgifter/arbetsgivaravgifter.4. 233f91f71260075abe8800020817.html. Accessed 15 Dec 2014

42. Van den Hout W. The value of productivity: human-capital versus friction-cost method. Annals of the rheumatic diseases. 2010;69(Suppl 1):i89-91.

\section{Submit your next manuscript to BioMed Central and we will help you at every step:}

- We accept pre-submission inquiries

- Our selector tool helps you to find the most relevant journal

- We provide round the clock customer support

- Convenient online submission

- Thorough peer review

- Inclusion in PubMed and all major indexing services

- Maximum visibility for your research

Submit your manuscript at www.biomedcentral.com/submit
Biomed Central 